Sādhanā Vol. 39, Part 3, June 2014, pp. 597-606. (C) Indian Academy of Sciences

\title{
Stability analysis of non-axisymmetric three-dimensional finite element rotor models with partial and full mass lumping
}

\author{
SMITADHI GANGULY ${ }^{1}$, A NANDI ${ }^{2, *}$ and $\mathrm{S} \mathrm{NEOGY}^{2}$ \\ ${ }^{1}$ Department of Mechanical Engineering, Hooghly Engineering and Technology \\ College, Hooghly 712 103, India \\ ${ }^{2}$ Department of Mechanical Engineering, Jadavpur University, \\ Kolkata 700 032, India \\ e-mail: nandi.arghya@gmail.com
}

MS received 26 September 2012; revised 21 January 2014; accepted 24 January 2014

\begin{abstract}
Unlike structural dynamics, the three-dimensional finite-element model of non-axisymmetric rotors on orthotropic bearings generates a large gyroscopic system with parametric stiffness. The present work explores the use of mass-lumping in stability analysis of such systems. Using a variant of Hill's method, the problem reduces to a generalized Eigen value problem of order $n m \times n m$, with $n$ as the order of the system in state vector representation and $m$ as the number of terms in the assumed solution. The matrices in both the sides of the Eigen value problem are expressed in terms of Kronecker products where the mass-matrix appears twice as a sub-matrix in both the sides of the equation. A particular one or both of them can be made diagonal. Both options produce sufficiently accurate results with considerable savings, even with a coarse mesh.
\end{abstract}

Keywords. Non-axisymmetric rotor; stability; Campbell diagram; threedimensional finite element; mass lumping.

\section{Introduction}

The research endeavor in this direction started with Rayleigh and Timoshenko beam elements (Nelson \& McVaugh 1976) for shaft and point inertia elements for discs. Though these elements are still very popular in practice, researchers extensively experimented with conical shaft elements, axisymmetric rotor elements and three-dimensional solid elements (Genta 1988, 1998; Nandi 2003; Nandi \& Neogy 2001). As the finite element models became more and more complicated over years, the number of degrees of freedom involved also increased manifold.

${ }^{*}$ For correspondence 
Stability analysis of three dimensional finite element models of 'non-axisymmetric' rotors has not been reported in literature. The present work makes a humble attempt in that direction. It further considers orthotropic flexible support which makes the stiffness matrix a parametric one. Then it examines the applicability of a standard lumping scheme in such an analysis.

In three-dimensional formulation one prefers a spinning frame for derivation of the governing equations (Nandi \& Neogy 2001). In this spinning frame, the orthotropic bearing stiffness becomes periodic. The governing equations thus become parametric in nature. A rotor crosssection is symmetric when the rotor has same bending stiffness in two perpendicular planes. A symmetric rotor on rigid, isotropic and orthotropic bearing is stable in absence of a destabilizing source like rotating damping, oil seals etc. A non-symmetric rotor is unstable in a specific region of spin speed (Kramer 1990).

The present work explores the possibility of using the HRZ lumping scheme (Cook et al 1989) in Eigen value analysis of three-dimensional finite element model of rotors. Though this lumping scheme is popular in structural dynamics, its use in stability analysis in rotor dynamics has not been reported in available literature. The shaft cross-sections considered are non-axisymmetric. For stability analysis of such non-axisymmetric rotors on orthotropic bearings, a variant of the Hill's method is adopted in this work (Nandi \& Neogy 2005). The final Eigen value problem is expressed in terms of Kronecker products. The mass matrix occurs twice in this expression. If a particular one of them is lumped, the whole Eigen value problem becomes a standard one. This case is referred to as partial lumping in the present work. The other one may be lumped or kept consistent. If both the occurrences are lumped, it is termed as full lumping. The partial and full lumping schemes mentioned above substantially reduce the number of operations and storage requirements. The interest lies here in finding out the accuracy of the results namely the Campbell diagram and the stability limits.

\section{Analysis}

\subsection{Rotor on orthotropic bearings}

In a spinning frame, the finite element equations for a three-dimensional rotor model supported on an orthotropic spring can be written asfollows (Nandi \& Neogy 2005):

$$
\left[M_{r}\right]\{\ddot{U}\}+\left[C_{r}\right]\{\ddot{U}\}+\left(\left[K_{r}\right]_{w b}+\left[K_{o}\right]_{b}+\left[K_{c}\right]_{b} \cos 2 \Omega t+\left[K_{s}\right]_{b} \sin 2 \Omega t\right)\{U\}=\{f\}
$$

$\left[M_{r}\right]$ Massmatrix.

$\left[C_{r}\right]$ Anti-symmetric Coriolis matrix.

$\left[K_{r}\right]_{w b}$ Stiffness matrix without considering the boundary (support) stiffness contributions

$\left[K_{o}\right]_{b}$ Matrix having non-zero contributions from support stiffness, which are not functions of time.

$\left[K_{c}\right]_{b},\left[K_{s}\right]_{b}$ Matrix having non-zero contributions from support stiffness terms, which are coefficients of cosine and sine functions respectively.

$\{f\}$ Excitation force on the rotor like unbalance, gravity etc.

The homogeneous part of eq. (1) is written in state vector form as follows: -

$$
[\bar{M}]\{\dot{Z}\}+[\bar{K}]\{Z\}+\left[\bar{K}_{c}\right] \cos 2 \Omega t\{Z\}+\left[\bar{K}_{s}\right] \sin 2 \Omega t\{Z\}=\{0\},
$$


where,

$$
\begin{aligned}
{[\bar{M}] } & =\left[\begin{array}{cc}
{\left[M_{r}\right]} & {[0]} \\
{[0]} & {[I]}
\end{array}\right] \\
{[\bar{K}] } & =\left[\begin{array}{cc}
{\left[C_{r}\right]} & {\left[K_{r}\right]_{w b}+\left[K_{o}\right]_{b}} \\
-[I] & {[0]}
\end{array}\right] \\
{\left[\bar{K}_{c}\right] } & =\left[\begin{array}{cc}
{[0]} & {\left[K_{c}\right]_{b}} \\
{[0]} & {[0]}
\end{array}\right] \\
{\left[\bar{K}_{s}\right] } & =\left[\begin{array}{cc}
{[0]} & {\left[K_{s}\right]_{b}} \\
{[0]} & {[0]}
\end{array}\right] .
\end{aligned}
$$

For rotor on orthotropic bearing, a solution of the eq. (2) can be assumed in the following form:

$$
\{Z\}=\{y(t)\} e^{\lambda t}
$$

where,

$$
\{y(t)\}=\left\{a_{0}\right\}+\left\{a_{2}\right\} \cos 2 \Omega t+\left\{b_{2}\right\} \sin 2 \Omega t+\left\{a_{4}\right\} \cos 4 \Omega t+\left\{b_{4}\right\} \sin 4 \Omega t+\ldots
$$

Substituting relation (4) in eq. (2) the following set of homogeneous first order ordinary differentials equations are obtained:

$$
[\bar{M}]\{y\}+\lambda[\bar{M}]\{y\}+[\bar{K}]\{y\}+\left[\bar{K}_{c}\right] \cos 2 \Omega t\{y\}+\left[\bar{K}_{s}\right] \sin 2 \Omega t\{y\}=\{0\} .
$$

If a five term solution is considered, a single element in the $\{y\}$ vector can be represented as

$$
y_{k}=a_{0}^{k}+a_{2}^{k} \cos 2 \Omega t+b_{2}^{k} \sin 2 \Omega t+a_{4}^{k} \cos 4 \Omega t+b_{4}^{k} \sin 4 \Omega t,
$$

This can be written as,

$$
y_{k}=[T]\left\{d^{k}\right\}=T_{j} d_{j}^{k}
$$

where,

$$
\begin{aligned}
{[T] } & =[1 \cos 2 \Omega t \sin 2 \Omega t \cos 4 \Omega t \sin 4 \Omega t] \\
\left\{d^{k}\right\} & =\left[a_{0}^{k} a_{2}^{k} b_{2}^{k} a_{4}^{k} b_{4}^{k}\right]^{T} .
\end{aligned}
$$

Now,

$$
\dot{y}_{k}=\dot{T}_{j} d_{j}^{k}=T_{i} D_{1_{i j}} d_{j}^{k},
$$

where, the matrix $\left[D_{1}\right]$ is the first order differentiation operation matrix.

Neglecting higher order terms, the product $y_{k} \cos 2 \Omega t$ can be expressed as follows:

$$
y_{k} \cos 2 \Omega t=T_{i} P_{c_{i j}} d_{j}^{k}
$$

where, the matrix $\left[P_{c}\right]$ is the product operation matrix for stiffness with cosine coefficients.

Similarly, the product $y_{k} \sin 2 \Omega t$ can be expressed as follows

$$
y_{k} \sin 2 \Omega t=T_{i} P_{s_{i j}} d_{j}^{k},
$$

where, the matrix $\left[P_{S}\right]$ is the product operation matrix for stiffnesses with sine coefficients. 
Eq. (6) can be expressed in indicial notation as follows

$$
\bar{m}_{i j} \dot{y}_{j}+\lambda \bar{m}_{i j} h_{j}+\bar{k}_{i j} y_{j}+\bar{k}_{c_{i j}} \cos 2 \Omega t y_{j}+\bar{k}_{s_{i j}} \sin 2 \Omega t y_{j}=0 .
$$

Replacing $y_{j}$ by its assumed solution as given in eq. (7) and making use of the first order differentiation operation matrix and the product operation matrices for cosine and sine terms, the following expression is obtained:-

$$
\bar{m}_{i j} T_{k} D_{1 k l} d_{l}^{j}+\lambda \bar{m}_{i j} T_{k} d_{k}^{j}+\bar{k}_{i j} T_{k} d_{k}^{j}+\bar{k}_{c_{i j}} T_{k} P_{c_{k l}} d_{l}^{j}+\bar{k}_{s_{i j}} T_{k} P_{s_{k l}} d_{l}^{j}=0,
$$

or,

$$
T_{k} m_{i j} D_{1_{k l}} d_{l}^{j}+\lambda T_{k} \bar{m}_{i j} \delta_{k l} d_{l}^{j}+T_{k} \bar{k}_{i j} \delta_{k l} d_{l}^{j}+T_{k} \bar{k}_{c_{i j}} P_{c_{k l}} d_{l}^{j}+T_{k} \bar{k}_{s_{i j}} P_{s_{k l}} d_{l}^{j}=0 .
$$

Finally one obtains,

$$
[A]\{X\}=-\lambda[B]\{X\},
$$

where,

$$
\begin{aligned}
& {[A]=\left([\bar{M}] \otimes\left[D_{1}\right]+[\bar{K}] \otimes[I]+\left[\overline{K_{c}}\right] \otimes\left[P_{c}\right]+\left[\overline{K_{s}}\right] \otimes\left[P_{s}\right]\right)} \\
& {[B]=[\bar{M}] \otimes[I]} \\
& \{X\}=\left\{\begin{array}{c}
\left\{d^{1}\right\} \\
\left\{d^{2}\right\} \\
- \\
-
\end{array}\right\} .
\end{aligned}
$$

The Kronecker product between two matrices $[A]$ and $[B]$ are defined as follows

$$
[A] \otimes[B]=\left[\begin{array}{ccc}
a_{11}[B] & a_{12}[B] & - \\
a_{21}[B] & a_{22}[B] & - \\
- & - & - \\
- & - & -
\end{array}\right] .
$$

\subsection{Partial lumping of mass matrix}

In partial mass lumping technique the mass matrix in the left hand side comes from a consistent formulation. The mass matrix in the right hand is a lumped one.

Thus in eq. (13) one uses a lumped mass matrix in the right hand side and a consistent one in the left.

The right hand side now becomes $\left[\begin{array}{cc}{\left[\left(m_{r}\right)_{i i}\right]} & {[0]} \\ {[0]} & {[I]}\end{array}\right] \otimes[I]=\left[(b)_{i i}\right]$

where, the sub-matrix $\left(m_{r}\right)_{i i}$ stands for the lumped mass matrix corresponding to the consistent mass matrix $\left(m_{r}\right)$ used in eq. (1).

Eq. (13) becomes

$$
\left[(1 / b)_{i i}\right][A]\{X\}=-\lambda\{X\}
$$

where, the matrices $\left[(b)_{i i}\right]$ and $\left[(1 / b)_{i i}\right]$ are both diagonal matrices. Diagonal element of one is the reciprocal of the corresponding one of the other. Therefore, the partial mass lumping converts the generalized Eigen value problem to a standard one. 


\subsection{Full lumping of mass matrix}

Another option is to lump the mass matrices in both the right and left hand sides. Now, the left hand side becomes

$$
[A]=\left(\left[\begin{array}{cc}
{\left[\left(m_{r}\right)_{i i}\right]} & {[0]} \\
{[0]} & {[I]}
\end{array}\right] \otimes\left[D_{1}\right]+[\bar{K}] \otimes[I]+\left[\bar{K}_{c}\right] \otimes\left[P_{c}\right]+\left[\bar{K}_{s}\right] \otimes\left[P_{s}\right]\right) .
$$

The right hand side remains same as follows

$$
\left[\begin{array}{cc}
{\left[\left(m_{r}\right)_{i i}\right]} & {[0]} \\
{[0]} & {[I]}
\end{array}\right] \otimes[I]=\left[(b)_{i i}\right] .
$$

The Eigen value problem now becomes

$$
\left[(1 / b)_{i i}\right][A]\{X\}=-\lambda\{X\} .
$$

This is referred to as full mass lumping in the present work.

\subsection{Stability analysis}

In linear stability analysis, the Eigen values are complex numbers whose imaginary parts are the whirl frequencies and the real parts decide stability. In rotor dynamics, the imaginary and real parts of the Eigen values are functions of spin speed. The plot of whirl frequencies against spin speed is the well-known Campbell diagram. A positive real part of an Eigen value indicates instability of that mode. In the present work the stability margin of the rotor model is determined from a plot of largest real part of the Eigen values versus spin speed.

\subsection{HRZ lumping scheme}

The HRZ lumping scheme is a simple and effective scheme (Cook et al 1989) for producing a diagonal mass matrix for structural dynamic analysis. The basic idea is to use the scaled diagonal terms of the consistent mass matrix. The scaling is so done that the total mass of the element is preserved. The scale factor is determined by dividing the total mass by the sum of the diagonal terms associated with a translational degree of freedom.

\section{Numerical study}

The present approach is extensively tested for a number of rotor configurations with rectangular, elliptical, tapered rectangular, tapered elliptical shafts. The results for two cases are presented here. In the first one a cantilevered rotor is considered with rectangular shaft and a disc at the free end. The second case considers a rectangular shaft with hinge support at the left end. The right end support is flexible and orthotropic.

Example 1. As mentioned before, a cantilevered steel rotor of length $l=400 \mathrm{~mm}$ is considered. The rectangular cross-section has sides of $b=20 \mathrm{~mm}$ and $h=30 \mathrm{~mm}$. The disc has a diameter of $d=100 \mathrm{~mm}$ and thickness $t=20 \mathrm{~mm}$. The finite element mesh is shown in figure 1a. This problem is a special case as the bearing at the fixed end is rigid. In rotor dynamics, a deep groove bearing is idealized as a fixed support. In a spinning frame, here the bearing stiffness does not 
(a)

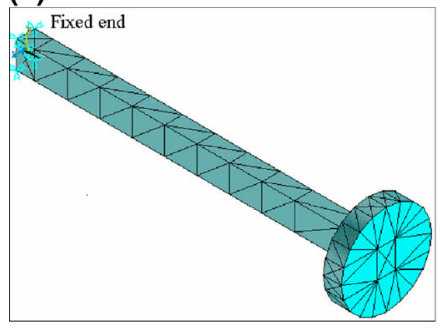

(d)

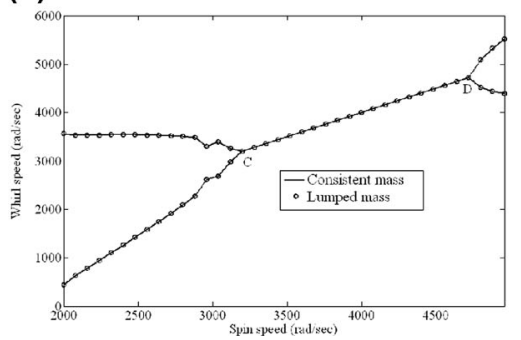

(b)

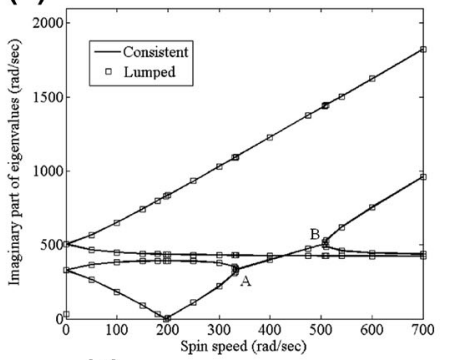

(e)

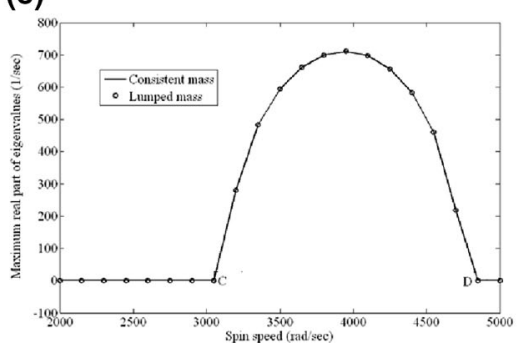

(c)

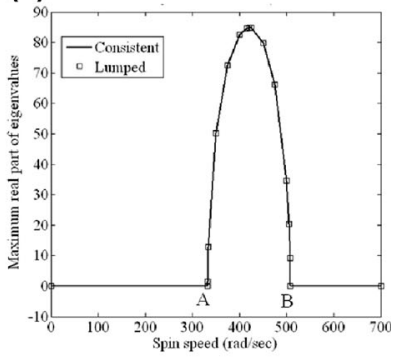

\section{列}


The instability starts where the largest real part suddenly shoots up to a positive value from zero. The onset of instability is marked by point $\mathrm{A}$ in the above figures. The unstable region ends at a spin speed when the largest real part again becomes zero. The rotor is unstable approximately between $330 \mathrm{rad} / \mathrm{s}$ and $510 \mathrm{rad} / \mathrm{s}$. The rotor is again stable beyond point $\mathrm{B}$.

The unstable behavior again appears in the second mode. Figure 1d and 1e show the imaginary and real parts of the next two Eigen values. Here, the instability occurs between points $\mathrm{C}$ and $\mathrm{D}$. The rotor is unstable between spin speeds 3200 and $4750 \mathrm{rad} / \mathrm{s}$.

The Eigen values are computed using both the consistent and lumped mass approaches. Excellent agreement is achieved even with a coarse mesh.

Example 2. Example 2 is a more general case. Here a rectangular rotor is considered. The length, breadth and width are $l=400 \mathrm{~mm}, b=20 \mathrm{~mm}$ and $w=30 \mathrm{~mm}$ respectively. The finite element mesh is shown in figure $2 \mathrm{a}$. The left end of the rotor is supported in a self-aligning bearing,

(a)

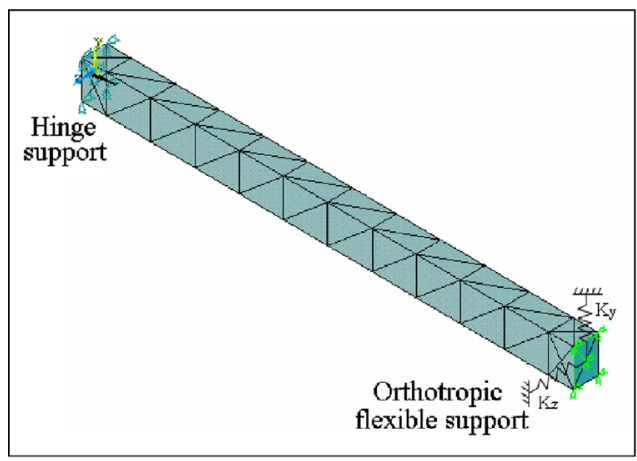

(b)

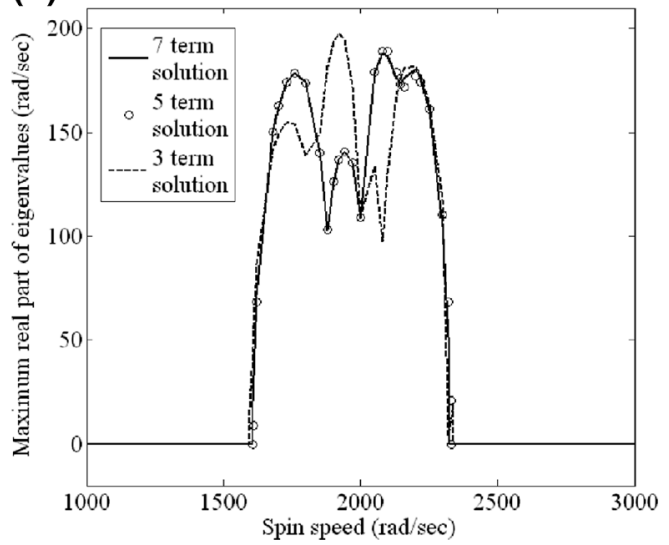

(c)

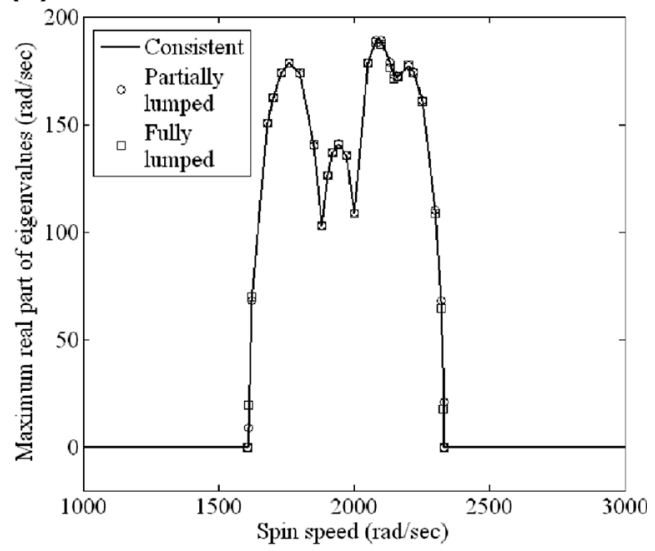

(d)

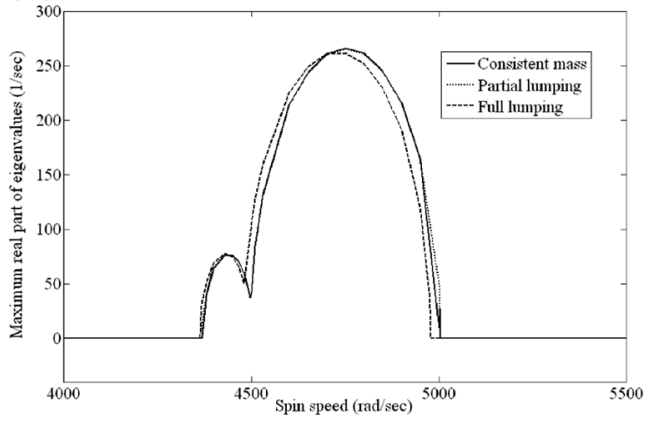

Figure 2. (a) Finite element mesh of a rectangular spinning shaft with self-aligning bearing at the left end and flexible support at the right (b) real parts of the Eigen values of a rectangular spinning shaft with self-aligning bearing at the left end and flexible support at the right, using 3, 5 and 7 terms in the assumed harmonic solution. (The fully lumped case is considered.) (c) real parts of a rectangular spinning shaft with self-aligning bearing at the left end and flexible support at the right using consistent, full and partial lumping considering 5 terms in the assumed harmonic solution (d) second zone of instability of a rectangular spinning shaft with self-aligning bearing at the left end and flexible support at the right. 
Table 1. The storage requirement and execution time for a sparse Eigen value analysis.

\begin{tabular}{lccc}
\hline & $\begin{array}{c}\text { No. of non-zero } \\
\text { elements in [B] }\end{array}$ & $\begin{array}{c}\text { No. of non-zero } \\
\text { elements in [A] }\end{array}$ & $\begin{array}{c}\text { CPU time for Eigen } \\
\text { value analysis (s) }\end{array}$ \\
\hline Consistent & 115970 & 497440 & 2.75 \\
Partial lumping & 10660 (Diagonal) & 497450 & 2.36 \\
Full lumping & 10660 (Diagonal) & 413192 & 1.92 \\
\hline
\end{tabular}

(a)

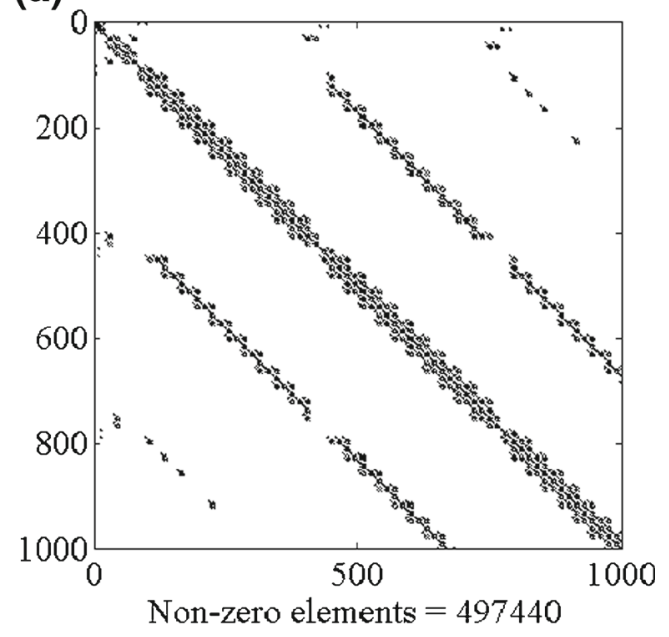

(b)

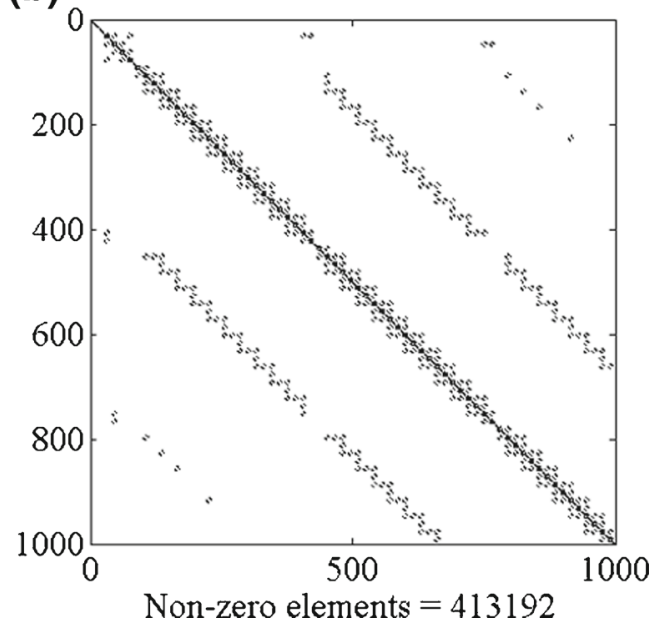

Figure 3. (a) Sparsity pattern of $[\mathrm{A}]$ matrix in case of consistent formulation - the contents of first thousand rows and columns are displayed (b) sparsity pattern of [A] matrix in case of full lumping - the contents of first thousand rows and columns are displayed. 
which can be idealized as a hinge. The right end is on a flexible orthotropic support. The support stiffness can be specified in an inertial reference as $k_{y y}=16000 \mathrm{~N} / \mathrm{mm}, k_{z z}=4000 \mathrm{~N} / \mathrm{mm}$. If the spin speed is denoted by the symbol $\Omega$, the support stiffness in a spinning frame can be represented as

$$
\left[\begin{array}{cc}
\cos \Omega t & \sin \Omega t \\
-\sin \Omega t & \cos \Omega t
\end{array}\right]^{T}\left[\begin{array}{cc}
k_{y y} & 0 \\
0 & k_{z z}
\end{array}\right]\left[\begin{array}{cc}
\cos \Omega t & \sin \Omega t \\
-\sin \Omega t & \cos \Omega t
\end{array}\right]
$$

Evidently, this contributes to a parametric stiffness term.

In the previous case, the problem is gyroscopic but not parametric. In this case the system is both gyroscopic and parametric. The finite element mesh is shown in figure $2 \mathrm{a}$. In order to formulate the matrix Eigen value problem one now requires the Hill's method. The Eigen value computation starts from eq. (13). The Eigen values are computed considering three, five and seven terms in the assumed solution. The computed real parts are compared in figure $2 \mathrm{~b}$. The five term and seven term solutions produce very close results. Therefore, a five term solution is found to be sufficient for the problem considered. The results obtained using consistent mass, partial lumping and full lumping are presented in figure $2 \mathrm{c}$. The first unstable region is between 1650 and $2300 \mathrm{rad} / \mathrm{s}$. There is again a second unstable region approximately between 4400 and $4900 \mathrm{rad} / \mathrm{s}$ (figure 2d). The results are in excellent agreement and are hardly distinguishable in the graphical representation of the first unstable region.

The sparse Eigen solver available in MATLAB is used in the present work. The number of non-zero elements in the matrices $[A]$ and $[B]$ for the problem considered is presented in table 1 . The execution time for one eigensolution is also displayed in table 1 . The results show that full lumping saves $30 \%$ computational time. Construction of a Campbell diagram or determination of stability limits requires hundreds of such Eigen solutions. Therefore, the total savings, though not appreciable for a single solution, is indeed significant for the intended purpose. The sparsity patterns of the matrix $[A]$ for consistent and fully lumped cases are shown in figure $3 a$ and $b$.

\section{Conclusion}

The present analysis starts with the governing equations of motion of three-dimensional finite element model of non-axisymmetric rotors on orthotropic bearings. In order to handle the antisymmetric gyroscopic matrix, the equations are cast in state vector form. Then a variant of Hill's method converts the parametric system to a generalized algebraic Eigen value problem of much larger size. The Eigen value problem is presented in terms of Kronecker products. The original mass matrix appears as sub-matrix twice at two different locations of the Eigen value problem. As a first case, a particular one of the two occurrences of the mass matrices is lumped. Then both the mass matrices are lumped. The stability limits are computed using consistent mass matrix and two above-mentioned approaches of lumping. For rigid or isotropic bearings, the stiffness matrix does not have parametric terms and the computations are simpler. In the two examples described here and in large number of other attempted cases, the results are consistently in excellent agreement even when a coarse mesh is used. Five harmonic terms in the assumed solution in Hill's method is found to be sufficient for the problem considered here. 


\section{References}

Cook R D, Malkus D S and Plesha M E 1989 Concepts and Applications of Finite Element Method. New York: John Wiley \& Sons

Genta G 1988 Whirling of unsymmetrical rotors: A finite element approach based on complex co-ordinates. J. Sound Vib. 124(1): 27-53

Genta G 1998 Vibration of Structures and Machines. New York: Springer

Kramer E 1990 Dynamics of Rotors and Foundations. Berlin: Springer

Nandi A 2003 On computation of response of a rotor in the deformed configuration using three-dimensional solid finite elements. Commun. Numer. Meth. Eng. 19(3): 179-195

Nandi A and Neogy S 2001 Modelling of rotors using three-dimensional solid finite elements. J. Strain Analysis for Engi. Design 36(4): 359-371

Nandi A and Neogy S 2005 An efficient scheme for stability analysis of finite element asymmetric rotor models in a rotating frame. Finite Elements Anal. Des. 41: 1343-1364

Nelson H D and McVaugh J M 1976 The dynamics of rotor-bearing systems using finite elements. ASME J. Eng. Ind. 98(2): 593-600 